\title{
The impact of disasters on cancer care in Lebanon
}

\author{
Fady El Karak¹, Elsa Rawadi*,1 (D), Joud Sawan ${ }^{1}$ \& Fady Gh Haddad ${ }^{1}$ (D) \\ ${ }^{1}$ Department of Hematology \& Oncology, Faculty of Medicine, Saint Joseph University, Beirut, Lebanon \\ *Author for correspondence: elsa.rawadi@hotmail.com
}

\begin{abstract}
"'In the event of a natural or manmade disaster, cancer patients must remain a priority because they need specialized management not only for the impact of the event but also for disruption in their cancer care."
\end{abstract}

First draft submitted: 9 September 2020; Accepted for publication: 20 October 2020; Published online: 5 January 2021

Keywords: cancer $\bullet$ cancer care $\bullet$ catastrophe $\bullet$ disaster $\bullet$ impact $\bullet$ patients

Cancer represents one of the most frequent scourges worldwide and one of the most common causes of death. Cancer patients are among the most medically fragile populations [1] and the most prone to be affected by any disturbances that undermine the healthcare system. These patients require continuous cancer care both during and after any disaster that may fall upon them or their healthcare system [2]. During the past 12 months, Lebanon has faced many dramatic events, ranging from an unprecedented economic decline with the devaluation of the national currency, to the COVID-19 pandemic that devastated the country, and finally the most recent explosion at the heart of Beirut that shattered the Lebanese capital. These tragic incidents have severely affected the lives of cancer patients.

By the end of 2019, the Lebanese pound started losing its value progressively, eventually reaching the fifth of its original value July 2020. This devaluation has negatively influenced the access of patients to cancer care. Despite the stability of the drug prices in the market, the purchasing power of the Lebanese population dropped significantly as the incomes fell and the unemployment rate increased, reaching $40 \%$ in 2020 . As a result of this crisis, patients witnessed a rise in the prices of complementary tests, consultations and hospitalizations fees. Additionally, the importation of drugs was also reduced, resulting in a shortage of different molecules. In practice, this translated into a decline in patients' access to anticancer therapies, particularly novel and expensive drugs, with a reliance on the classical, older and less effective medications for treatment. Complementary tests and diagnostic procedures such as imaging, blood tests, screening modalities and interventions also became less affordable.

On 21 February 2020, the first case of COVID-19 was declared on the Lebanese territory, imported from Iran. By 22 August 2020, the total number of cases had reached 12,191, with a death toll of 121 [3]. The increase in the incidence of coronavirus infections has affected several aspects of cancer care, and the Lebanese Society of Medical Oncology issued a statement on the care of cancer patients during the COVID-19 pandemic [4]. Similar to what has been observed in other countries [5], the pandemic resulted in significant declines in the rates of screening, surgeries and follow-up visits. For instance, scheduled mammograms were deferred for later dates, screening colonoscopies were postponed, and elective surgeries were delayed by fear of viral transmission in the operation room. Moreover, a significant number of patients cancelled their regular follow-up visits with their physician by fear of contracting the coronavirus. The combination of these factors led to a reduction in the number of new cancer diagnoses, and as a result, it is postulated that the upcoming months will witness an increase in the number of new cases discovered at advanced stages.

Recently, on 4 August 2020, a massive explosion shook the heart of the capital, at the Beirut port. This disastrous incident was thought to be related to the detonation of a stock of ammonium nitrate stored in the port area. The blast sent a shockwave that severely damaged the surroundings in a 2-mile radius. One week after the explosion, the death toll has reached 220, with 110 people missing, approximately 6000 people injured and more than 300,000 
people left without shelter. Innumerable buildings, shops, establishments and hospitals were affected. In Beirut, three major hospitals were completely destroyed and subsequently had to shut down and relocate their patients to any available hospital capable of sustaining them. Three other hospitals were severely damaged and functioning well below their normal capacity but were nonetheless able to ensure adequate care for the victims. There has not been yet a final assessment of the damage, but according to the WHO's regional emergency director, the primary numbers show that more than 500 hospital beds have been lost and more than $50 \%$ of the capital's clinics and health centers are currently nonfunctional.

Cancer patients were among the populations that were heavily affected by this tragedy. Those requiring anticancer therapies at the damaged hospitals were forced to shift their care to other cancer centers. Hospitalization and chemotherapy units were also damaged in many hospitals of the capital, Beirut, where the majority of the country's cancer patients receive their treatments. As an example, the same-day chemotherapy unit at the Hotel-Dieu de France university hospital of Beirut was among the most damaged sections of the hospital. Consequently, hundreds of cancer patients were left without treatment for 4 days and either had to postpone their treatment or relocate to another center. In addition, approximately half of cancer patients in Lebanon have free access to cancer drugs via the ministry of public health and the governmental hospital of Quarantina. The latter, which has a large number of patients who rely on it as their sole source of medications, was heavily damaged by the blast and thus was unable to deliver the medications over a period of several days before progressively resuming activity. Also, a significant number of cancer drugs were stocked in a warehouse next to the explosion site and were therefore destroyed, making the access to various medications more challenging [6]. In addition, after disasters, the response within the community becomes focused and oriented mainly toward providing acute care for the injured, and access to treatment and medication for people with chronic diseases, such as cancer, becomes endangered [7]. Rapid-response teams deployed to disaster areas should be trained to implement essential noncommunicable disease interventions and to include essential cancer medications in emergency kits. In addition, despite their significant assistance for the victims, humanitarian organizations are offering limited coverage for the management of cancer because of limited resources, other more urgent priorities and high costs. Thus, the global health community must change its vision, approach and strategy to tackle this issue.

Another impact of this tragic event is that after the explosion, hygiene and protective measures to prevent the spread of COVID-19, such as wearing a facial mask and social distancing, lost priority among the chaos. The streets of Beirut, as well as the medical facilities in the city, were overcrowded with the wounded, their relatives and the healthcare workers. This was reflected by a surge in the number of COVID-19 cases that sharply increased from 209 cases on the day of the explosion to more than 500-600 daily cases in the weeks after the blast. Without doubt, it will have numerous consequences on the rate of hospitalizations and on cancer patients who are considered a fragile and immunocompromised population.

In the event of a natural or manmade disaster, cancer patients must remain a priority because they need specialized management not only for the impact of the event but also for disruption in their cancer care. This requires planning on three levels: with their healthcare provider, with their family and surroundings and on a personal level. The role of the physician is to provide patients with a clear treatment schedule accommodating for disruptions, a list of drugs to treat any possible side effects of the medication and a short, detailed description of the patient's case provided this is needed due to patient translocation. It is important that patients be well informed about their disease and how it might be affected by the crisis (treatment delay, delay in imaging if the equipment was damaged, reduction of hospital capacity for admitting patients suffering from side effects, etc.), the availability of different medications in the healthcare center or in the country, as well as to the precautions to be taken to avoid infection with COVID-19. If the patient is not knowledgeable about his or her disease or unable of perform self-care, the responsibility falls on a family member or close caregiver. Another role for the family members and the physician is to provide psychosocial care for the patient in case of trauma and to prioritize patient needs in accordance with his or her condition - most importantly, pharmaceutical needs, followed by dietary, supportive and physical therapy needs.

Currently, the WHO is collaborating with the Lebanese Ministry of Public Health to develop a plan and raise funding to coordinate the restoration of damaged health facilities and maintain a sufficient supply of medicine as part of its Beirut Port Blast Emergency Strategic Response Plan [8]. In light of the country's difficult economic situation, implementation of this plan may be delayed, and the expected results may be hard to achieve. This may have a negative impact on cancer patients in more ways than one, such as any shortage in treatment supply or any delay that may worsen their disease outcome and prognosis. For instance, many third-party payers and hospitals have 
already stopped approving and supplying the more expensive therapies, including immunotherapy and targeted therapies, thus forcing the physicians to resort to older protocols and less expensive, traditional chemotherapy drugs. However, other countries and private international institutions have shown solidarity with Lebanese cancer patients. In fact, after the explosion, humanitarian assistance was provided specifically for cancer patients, in the form of medications, hospital material required for the management of cancer patients, funding dedicated to the restoration or relocation of damaged oncology units; some institutions outside of Lebanon also offered care abroad to some cancer patients.

In conclusion, disasters and economic problems can negatively influence care for cancer patients, who represent a fragile population. In Lebanon, cancer patients were severely affected over the past year by the negative consequences of the economic crisis, the COVID-19 pandemic and the massive Beirut explosion. Efforts should be made to provide rapid access to medications for these patients as well as relocation of their care to other cancer centers to spare these patients additional burden.

\section{Financial \& competing interests disclosure}

The authors have no relevant affiliations or financial involvement with any organization or entity with a financial interest in or financial conflict with the subject matter or materials discussed in the manuscript. This includes employment, consultancies, honoraria, stock ownership or options, expert testimony, grants or patents received or pending, or royalties.

No writing assistance was utilized in the production of this manuscript.

\section{References}

1. Rodriguez-Rabassa M, Hernandez R, Rodriguez Z et al. Impact of a natural disaster on access to care and biopsychosocial outcomes among Hispanic/Latino cancer survivors. Sci. Rep. 10(1), 10376 (2020).

2. Gorji HA, Jafari H, Heidari M, Seifi B. Cancer patients during and after natural and man-made disasters: a systematic review. Asian Pac. J. Cancer Prev. 19(10), 2695-2700 (2018).

3. Ministry of Public Health in Lebanon. www.moph.gov.lb

4. Bitar N, Kattan J, Kourie HR, et al. The Lebanese Society of Medical Oncology (LSMO) statement on the care of patients with cancer during the COVID-19 pandemic. Future Oncol. 16(11), 615-617 (2020).

5. Yin K, Singh P, Drohan B, Hughes KS. Breast imaging, breast surgery, and cancer genetics in the age of COVID-19. Cancer 126(20), 4466-4472 (2020).

6. Gourd E. Patients with cancer hit hard by deadly explosions in Beirut. Lancet Oncol. 21(9), 1143 (2020).

7. El Saghir NS, Soto Pérez de Celis E, Fares JE, Sullivan R. Cancer care for refugees and displaced populations: Middle East conflicts and global natural disasters. Am. Soc. Clin. Oncol. Educ. Book 38, 433-440 (2018).

8. WorldHealth Organization. Beirut port blast, emergency strategic response plan. (2020).

http://www.emro.who.int/images/stories/lebanon/who-lebanon-strategic-response-plan-27.9.20.pdf?ua=1 